\title{
T1-Weighted Dynamic Contrast-Enhanced MRI Is a Noninvasive Marker of Epidermal Growth Factor Receptor vIII Status in Cancer Stem Cell-Derived Experimental Glioblastomas
}

W e read with great interest the article by Arevalo-Perez et al, ${ }^{1}$ describing the potential value of T1-weighted dynamic contrast-enhanced MR imaging (DCE-MR imaging) as a biomarker for epidermal growth factor receptor variant III (EGFRvIII) mutation in patients with glioblastoma (GBM). In this retrospective study, the authors showed that EGFRvIII-positive tumors, compared with GBMs lacking the mutation, presented with a statistically significant increase in perfusion values of the contrast transfer coefficient $\left(K^{\text {trans }}\right)$ and plasma volume $(\mathrm{Vp})$. We agree with the authors' findings, and we also share some of their concerns; as the authors clearly stated in their discussion, both the genetic test performed on the tumor samples and the biopsy location may have induced some bias in their results. Specifically, the genetic test did not account for other mutations on the EGFR gene, which may also correlate to altered perfusion values; and the lack of the precise stereotactic biopsy localization may have confounded the assessment of EGFRvIII status. Furthermore, in this retrospective work, the possible presence of other concomitant mutations of genes regulating the tumor vascularization was not ruled out and might have contributed to the increased permeability of tumors positive for EGFRvIII.

To substantiate their findings, we tested the authors' hypothesis on a preclinical rat model of cancer stem cell (CSC)-derived GBM. CSCs were isolated by the neurosphere assay from a primary GBM tissue specimen, which was negative for the EGFRvIII mutation. CSCs were transduced with a lentiviral vector, coding for either the green fluorescent protein (GFP) or EGFRvIII under a constitutive promoter, according to our previously published protocol. ${ }^{2}$ EGFRvIII messenger RNA and protein expression was confirmed by real-time polymerase chain reaction and Western blot, respectively. Five $\times 10^{5}$ transduced GBM CSCs were then injected into the left striatum of Rowett Nude rats; 3 rats were injected with GFP-transduced CSCs, whereas 4 rats were injected with the EGFRvIII-transduced CSCs.

MR imaging was performed on a small animal-dedicated 7T scanner (30/70 BioSpec; Bruker, Ettlingen, Germany) 35 days after CSC injection. The protocol included DCE-MR imaging during injection of gadobutrol (Gadavist; Bayer Schering Pharma, Berlin, Germany) performed by using a dynamic gradient-echo T1-weighted sequence $\left(\mathrm{TR} / \mathrm{TE}=67 / 3 \mathrm{~ms}\right.$, flip angle $=30^{\circ}$, matrix $=170 \times 170$,

http://dx.doi.org/10.3174/ajnr.A4774 in-plane voxel size $=0.21 \mathrm{~mm}^{2}$, section thickness $=0.75 \mathrm{~mm}$ ). Eighty dynamic scans were obtained. DCE-MR imaging was preceded by a saturation recovery sequence for T1 mapping and followed by a contrast-enhanced $\mathrm{T} 1$ sequence for anatomic reference. DCE-MR imaging data were processed by using the software nordicICE (NordicNeuroLab, Bergen, Norway). Maps of pharmacokinetic parameters, $\mathrm{Vp}$ and $K^{\text {trans }}$, were calculated after deconvolution with an averaged arterial input function. Quantitative values were then obtained from both the region of maximal abnormality (hotspots) and histogram analysis. Volumes of interest were drawn on contrast T1-weighted images, previously coregistered to the DCE-MR imaging parametric maps. Values from both hotspots and histogram analysis were then normalized by using the ratio of tumor to normal white matter by placing ROIs on the contralateral healthy hemisphere, consistent with the analysis performed in our reference study. ${ }^{1}$

As shown in Fig 1, our results confirm the observations by Arevalo-Perez et al $^{1}$ : in fact, EGFRvIII-transduced CSC-derived tumors presented as very large lesions with heterogeneous contrast enhancement and a marked increase in tumor volume compared with the control GFP-transduced CSC-derived lesions. The mean volume for GFP-transduced CSC-derived lesions was $77.96 \mathrm{~mm}^{3}$ (range, 41.48-131.96 $\mathrm{mm}^{3}$ ), whereas for EGFRvIII-transduced CSC-derived tumors, the mean volume was $279.35 \mathrm{~mm}^{3}$ (246.82-331.35 $\mathrm{mm}^{3}$ ). Moreover, the latter tumors displayed markedly increased mean values of both normalized $K^{\text {trans }}$ and $\mathrm{Vp}$ in the hotspot analysis, respectively 38.41 (range, 15.2-64.4) and 18.51 (9.2-26.7), compared with mean values of $6.77(5.8-7.5)$ and $4.88(2.6-7.5)$ for the control group. Histogram analysis in EGFRvIII-transduced CSC-derived tumors also showed an increase of the mean 75th and 90th percentile values for both $K^{\text {trans }}$ and Vp. In EGFRvIII-transduced CSC-derived tumors, the mean normalized (relative) values were, respectively, 4.91 (range, 3.1-10.5), 5.22 (3.2-12.1) (75th), and 4.73 (2.5-10.9) (90th) for $K^{\text {trans }}$ and 3.05 (2.8-4.9), 2.4 (2.2-4.2), and 2.43 (2.1-4.4) for Vp. For GFP-transduced CSC-derived lesions, the normalized values for $K^{\text {trans }}$ were $1.91(1.2-3.4), 3.42(1.2-3.4)$, and $3.13(1.4-3.3)$ and they were 2.68 (3.0-4.1), 2.12 (2.7-3.4), and 2.05 (1.7-3.1) for Vp. Immunofluorescence analysis of tumors confirmed an increase of vascularization in the brains transplanted with the EGFRvIII-transduced CSCs.

In conclusion, our findings, showing that the constitutive ex- 

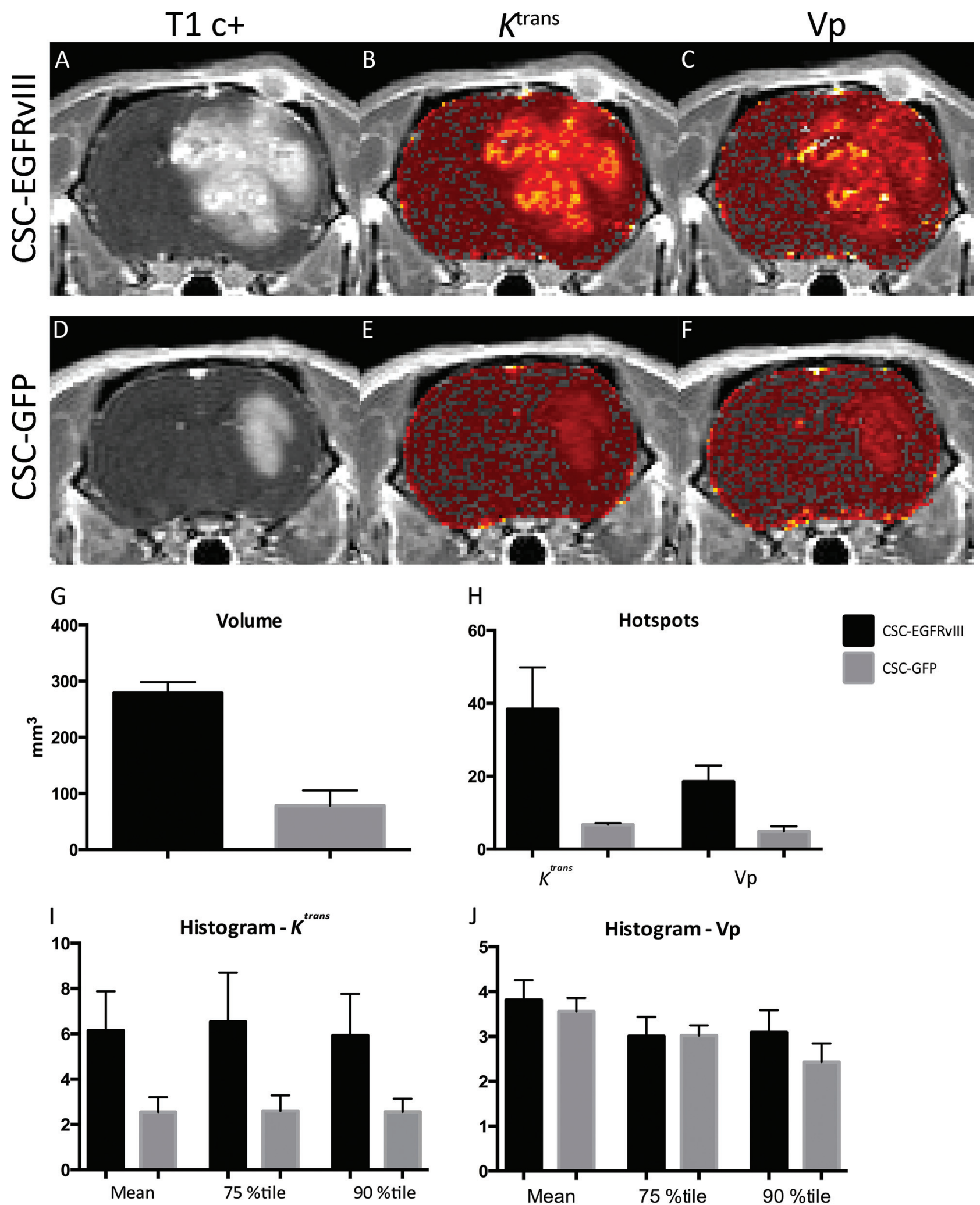

FIG 1. Representative postcontrast T1 and DCE-MR imaging parametric maps from EGFRvIll-transduced CSC-derived lesions ( $A-C)$ and control GFP-transduced CSC-derived lesions $(D-F)$. Axial contrast T1-weighted images $(A$ and $D)$ demonstrate a larger and more heterogeneously enhancing lesion in EGFRvIll-transduced CSC-derived tumors. Corresponding $K^{\text {trans }}$ and $V p$ maps reveal increased leakiness $(B)$ and increased perfusion (C) in EGFRvill-transduced CSC-derived lesions with respect to control lesions, which show little increase in $K^{\text {trans }}(E)$ or $V p(F)$. Each parametric map is displayed by using the same thresholds for both cell lines (range: $K^{\text {trans }}, 0-0.04$ minutes ${ }^{-1} ;$ range: $V p, 0-4$ ). G-J. Bar graphs show the mean and standard error for volume $(G)$, hotspots $(H)$, histogram normalized (relative) $K^{\text {trans }}(I)$, and normalized $V p(l)$ values in CSC-EGFRvIll (black bars) and CSC-GFP (gray bars) cell lines. 
pression of EGFRvIII in a human CSC line can determine a significant increase in tumor perfusion parameters in experimental GBM, strongly support the hypothesis formulated by Arevalo-Perez et al. ${ }^{1}$ Thus, the increase of permeability metrics may truly reflect the EGFRvIII status.

Disclosures: Marcello Cadioli-UNRELATED: Employment: Philips Healthcare. Rossella Galli-UNRELATED: Grants/Grants Pending: Associazione Italiana per la Ricerca sul Cancro (AIRC) Foundation, ${ }^{*}$ Comments: grant support from the Italian charitable foundation supporting cancer research. *Money paid to the institution.

\section{REFERENCES}

1. Arevalo-Perez J, Thomas AA, Kaley T, et al. T1-weighted dynamic contrast-enhanced MRI as a noninvasive biomarker of epidermal growth factor receptor vIII status. AJNR Am J Neuroradiol 2015;36:2256-61 CrossRef Medline

2. Mazzoleni S, Politi LS, Pala M, et al. Epidermal growth factor receptor expression identifies functionally and molecularly distinct tumor-initiating cells in human glioblastoma multiforme and is required for gliomagenesis. Cancer Res 2010;70:7500-13 CrossRef Medline

(D) L.S. Politi

Neuroimaging Research, Hematology/Oncology Division Boston Children's Hospital/Dana Farber Cancer Institute Boston, Massachusetts Radiology Department University of Massachusetts Medical School Worcester, Massachusetts
Neuroradiology Unit and CERMAC

Vita-Salute San Raffaele University and IRCCS San Raffaele Scientific Institute

Milan, Italy

(1) G. Brugnara

(1) A. Castellano

(D) M. Cadioli

(D. Altabella

Neuroradiology Unit and CERMAC

Vita-Salute San Raffaele University and IRCCS San Raffaele Scientific Institute

Milan, Italy

(D) M. Peviani

Neuroimaging Research, Hematology/Oncology Division Boston Children's Hospital/Dana Farber Cancer Institute Boston, Massachusetts

Neuroradiology Unit and CERMAC

Vita-Salute San Raffaele University and IRCCS San Raffaele Scientific Institute

Milan, Italy

(D) Mazzoleni

Neural Stem Cell Biology Unit, Division of Regenerative Medicine, Stem Cells and

Gene Therapy

IRCCS San Raffaele Scientific Institute

Milan, Italy

(1) A. Falini

Neuroradiology Unit and CERMAC

Vita-Salute San Raffaele University and IRCCS San Raffaele Scientific Institute

Milan, Italy

(D) R. Galli

Neural Stem Cell Biology Unit, Division of Regenerative Medicine, Stem Cells and

Gene Therapy

IRCCS San Raffaele Scientific Institute,

Milan, Italy 